\title{
A Virtual Element Model for the prediction of long-term salt marsh dynamics
}

\author{
$\underline{\text { M. Ferronato }}^{\text {a }}{ }^{\text {, A. Mazzia, }}{ }^{\text {a }}$, P. Teatini, ${ }^{\text {a }}$, C. Zoccarato ${ }^{a}$ \\ ${ }^{a}$ Department of Civil, Environmental and Architectural Engineering, University of Padova, Italy \\ Email: massimiliano.ferronato@unipd.it
}

\begin{abstract}
Salt marshes are vulnerable environments hosting complex interactions between physical and biological processes. The prediction of long-term vertical dynamics, i.e., marsh growth and/or reduction, is crucial to estimate the potential impacts of different forcing scenarios on such systems. The most significant processes influencing the elevation of the salt-marsh platform are accretion, auto-compaction, and the variation rates of the relative sea level rise, i.e., land subsidence of the marsh basement and eustatic rise of the sea level. The accretion term considers the vertical sedimentation of organic and inorganic material over the marsh surface, whereas the compaction reflects the progressive consolidation of the porous medium under the increasing load of the overlying younger deposits. The present work describes a novel mathematical approach, based on the Virtual Element Method, for the long-term simulation of the salt marsh vertical dynamics. The Virtual Element approach is a grid-based variational technique for the numerical discretization of Partial Differential Equations allowing for the use of very irregular meshes consisting of a free combination of different polyhedral elements. The modelling approach provides the pore pressure evolution within a compacting/accreting vertical cross-section of the marsh, coupled to a geomechanical module based on Terzaghi's principle of effective inter-granular stress. The model takes into account the geometric non-linearity caused by the large salt marsh deformations by using a Lagrangian approach with an adaptive grid, where the domain geometry changes in time to follow the deposit consolidation and the new sedimentation. The use of Virtual Elements ensures a great flexibility in the element generation and management, avoiding the numerical issues often arising from strongly distorted meshes. The numerical model is developed, implemented and tested employing two different configurations of the sedimentation $r$ ate. The preliminary numerical results provide evidence of the flexibility of the proposed approach, which appears to be a promising computational tool for the accurate simulation of real-world applications.
\end{abstract}

Keywords: Salt marshes, long-term dynamics, numerical modeling, virtual element method 


\section{INTRODUCTION}

The accretion/regression of the surface elevation of a marshland is a complex process hosting the interaction of different mechanisms. Typically, the active sediment deposition and compaction rate are the most important driving factors, along with the possible presence of external forcing actors such as the relative sea level rise (RSLR), the tidal and wave regimes, and the erosion (Allen, 2000; Morris et al., 2002). Since the geomorphological equilibrium of coastal wetlands is regulated by the interaction of sea level, land elevation, primary production and sediment accretion (Morris et al., 2002), the analysis of the salt marsh stratigraphies plays a key role for the reconstruction of the sea-level evolution and the deposition history. Hence, the accurate estimate of the marsh compaction is extremely important for a correct interpretation of the sea-level evolution, thus avoiding its overestimation (Brain et al., 2011). However, despite its importance, the compaction term is often computed with the aid of very simplified approaches, such as empirical relationships (Allen, 1999; Sheldon and Retallack, 2001; Skempton, 1970), 1D compaction models (Brain et al., 2011; Paul and Barras, 1998; Massey et al., 2006), or solving the 1D groundwater flow equation based on the standard Darcy's law (Pizzuto and Schwendt, 1997; Kooi and de Vries, 1998).

Due to the extremely high porosity of newly deposited material, in the range of 0.5-0.9 (Brain et al., 2011), the medium is highly deformable. In this paper, the large solid grain motion is accounted for by recasting Darcy's law in terms of relative velocity of fluid to grains. The porous matrix undergoes a progressive 1D vertical consolidation under the effect of an increasing load due to the new deposition of sediments and the related overpressure dissipation.

The present work describes a novel mathematical approach, based on the Virtual Element Method (VEM), for the long-term simulation of the salt marsh vertical dynamics. The VEM approach is a grid-based variational technique for the numerical discretization of PDEs allowing for the use of very irregular meshes consisting of a free combination of different polyhedral elements. The modeling approach provides the pore pressure evolution within a compacting/accreting vertical cross-section of the marsh, coupled to a geomechanical module based on Terzaghi's principle of effective intergranular stress. Soil properties, such as porosity, permeability, and compressibility, vary with the effective intergranular stress according to empirical non-linear constitutive relationships. The model takes into account the geometric non-linearity caused by the large salt marsh deformations by using a Lagrangian approach with an adaptive grid, where the domain geometry changes in time to follow the deposit consolidation and the new sedimentation. The use of the VEM approach for integrating the governing PDEs ensures a great flexibility in the element generation and management, avoiding the numerical difficulties arising from strongly distorted meshes.

The effectiveness and potential of the VEM approach is investigated in two preliminary test cases, where the numerical model is used to simulate the vertical marsh evolution in a time lapse of hundreds of years. In particular, two loading conditions are considered, thus leading to a different evolution of the computational grid. The paper is organized as follows. Section 2 and 3 describe the PDEs governing the groundwater flow and the porous medium compaction modules, along with their numerical discretization using the VEM approach. The model set-up and the preliminary results obtained with the proposed modeling framework are described and discussed in Section 4. A few concluding remarks close the presentation.

\section{GROUNDWATER FLOW AND COMPACTION MODELS}

The rigorous formulation of the PDE governing the 1D flow in an elastic saturated porous medium subjected to large deformations was originally proposed by Gambolati (1973a,b), where the hypothesis of infinitesimal displacements of the solid grains is relaxed by the introduction of a geometric non-linearity. Considering a 2D domain undergoing a vertical (1D) compaction/accretion process, the groundwater flow equation can be written as (Zoccarato and Teatini, 2017):

$$
\frac{\partial}{\partial x}\left(\frac{k_{x}}{\gamma} \frac{\partial p}{\partial x}\right)+\frac{\partial}{\partial z}\left(\frac{k_{z}}{\gamma} \frac{\partial p}{\partial z}\right)=\left(c_{b}+\phi \beta\right) D p-2 \beta k_{z} \frac{\partial p}{\partial z}-\beta \frac{k_{x}}{\gamma}\left(\frac{\partial p}{\partial x}\right)^{2}-\beta \frac{k_{z}}{\gamma}\left(\frac{\partial p}{\partial z}\right)^{2}
$$

where $k_{x}$ and $k_{z}$ are the horizontal and vertical hydraulic conductivities, $\gamma$ is the specific weight of water, $c_{b}$ is the soil oedometric compressibility, $\phi$ is the soil matrix porosity, $\beta$ is the volumetric water compressibility, $p$ is the incremental pore pressure with reference to the hydrostatic condition, $x$ and $z$ are the horizontal and vertical coordinates, $t$ is time, and $D$ is total or Eulerian derivative:

$$
D=\frac{\partial p}{\partial t}+v_{g, z} \frac{\partial p}{\partial z}
$$


with $v_{g, z}$ the vertical grain velocity.

Equation (1) holds true under the following assumptions: (i) $k_{x}$ and $k_{z}$ are equal to $k_{x}^{*} \gamma / \mu$ and $k_{z}^{*} \gamma / \mu$, respectively, with constant values for $k_{x}^{*}, k_{z}^{*}$, and $\mu$, i.e., the medium intrinsic horizontal and vertical permeability and the water viscosity; (ii) $\gamma=\gamma_{0} \exp \left[\beta\left(p_{0}+p\right)\right]$, where $\gamma_{0}$ and $p_{0}$ are initial reference values for $\gamma$ and $p$, respectively; (iii) the relative Darcy's law can be applied:

$$
\phi\left(v_{w, i}-v_{g, i}\right)=-k_{i} \frac{\partial \psi}{\partial i}, \quad i=x, z
$$

with $v_{g, i}$ and $v_{w, i}$ the (absolute) velocity of solid grains and water along the $i$ direction, respectively, and $\psi$ the hydraulic head expressed as $\psi=z+\int_{0}^{p} \frac{d p}{\gamma}$. Notice that for the specific process of interest, $v_{g, x}$ is assumed to be negligible and only a vertical grain motion is considered; (iv) incompressible solid grains; and (v) the total stress is constant and expressed by Terzaghi's principle in the form $\sigma_{t}=\sigma_{z, 0}+\sigma_{z}+p_{0}+p$, with $\sigma_{z, 0}$ and $\sigma_{z}$ the initial and incremental intergranular effective stress, respectively.

The oedometric compressibility $c_{b}$ can be obtained by laboratory tests on soil samples and is related to the classical compressibility, $\alpha$, by (Gambolati et al., 1998):

$$
c_{b}=\frac{p \frac{d \alpha}{d p}+\alpha}{1+\alpha p}
$$

Thus, $c_{b}$ and $\alpha$ coincide when $p$ tends to zero.

The soil matrix compaction, $u(z, t)$, and the grain velocity, $v_{g}(z, t)$, are given by (Gambolati et al., 1998):

$$
\begin{aligned}
& u(z, t)=-\int_{0}^{z} \frac{\alpha \sigma_{z}}{1-\alpha \sigma_{z}} d z \\
& v_{g, z}(z, t)=\left(1-\alpha \sigma_{z}\right) \int_{0}^{z} \frac{\left(\alpha+\sigma_{z} \frac{\partial p}{\partial t}\right)}{\left(1-\alpha \sigma_{z}\right)^{2}} d z
\end{aligned}
$$

To account for the organic/inorganic sediment deposition over the marsh surface, a sedimentation rate, $\omega(x, t)$, is prescribed and, consequently, the total stress $\sigma_{t}$ of the compacting column is no longer constant as assumed in equation (1). Introducing equation (5) into (1) and neglecting the second-order terms yields (Zoccarato and Teatini, 2017):

$$
\frac{\partial}{\partial x}\left(\frac{k_{x}}{\gamma} \frac{\partial p}{\partial x}\right)+\frac{\partial}{\partial z}\left(\frac{k_{z}}{\gamma} \frac{\partial p}{\partial z}\right)=\left(\frac{\sigma_{z} \frac{d \alpha}{d \sigma_{z}}+\alpha}{1-\alpha \sigma_{z}}+\phi \beta\right) D p-\frac{\sigma_{z} \frac{d \alpha}{d \sigma_{z}}+\alpha}{1-\alpha \sigma_{z}} D \sigma_{t}
$$

In natural conditions, the variation of the total stress is due to the change of load caused by sediment deposition over the marsh surface:

$$
D \sigma_{t}=\omega\left(1-\phi_{0}\right)\left(\gamma_{s}-\gamma\right)
$$

with $\gamma_{s}$ the specific weight of the grains and $\phi_{0}$ the initial porosity at $\sigma_{z, 0}$.

Equation (6), equipped with appropriate initial and boundary conditions, governs the 2D groundwater flow in a cross-section of a deforming porous medium. The resulting incremental pore pressure distribution is then used in equation (4) to compute the current increment of vertical effective stress and predict the medium deformation.

\section{NUMERICAL MODEL}

The numerical solution of equations (4) and (6) is obtained by using a Lagrangian approach (Zoccarato and Teatini, 2017), where a dynamic mesh is employed and the grid nodes follow the grains during the consolidation process. In this way, over a moving node the Eulerian derivative $D p$ can be treated as a partial time derivative $\partial p / \partial t$.

The discretization of the governing equations is performed with the aid of the Virtual Element Method (VEM). The VEM approach is a novel numerical technque that can be regarded as a generalization of the classical 
conforming Finite Element Method (FEM) to general polygonal and polyhedral elements. At the same time, many theoretical aspects of VEM are intimately connected to Mimetic Finite Differences (MFD), so that the VEM can be also viewed as the ultimate MFD evolution able to preserve the attractive features with a greater ease of implementation. For many problems, the resulting systems of discrete equations are the same as MFD and, for a particular element choice, also FEM. In general, however, VEM offers additional possibilities with respect to FEM and MFD.

The basic VEM idea relies on selecting the approximation spaces and the degrees of freedom in such a way that the elementary stiffness matrix can be computed knowing the shape functions only on the boundary of each element. The VEM has been introduced quite recently (da Veiga et al., 2013), nevertheless several works already highlight the main theoretical properties and practical details on its implementation, e.g. da Veiga et al. (2014), Sutton (2016), and references therein. However, very few applications have been developed so far to real-world engineering and environmental problems. In particular, to our knowledge this is the first time VEM is used for the solution of the salt marsh vertical dynamics.

To briefly describe the low order VEM, we start from a decomposition of the PDE domain into general polygonal elements whose boundaries are not self-intersecting. As unknowns, we take the values of the approximating function at the vertices of the elements. For an elliptic differential operator, the weak variational form of the PDE uniquely defines on each element the approximating function through a linear interpolation of the nodal values along the element boundary and a harmonic extension inside the element. To avoid the local solution of a Laplace problem on an element-by-element basis, a different procedure is introduced in order to ensure consistency and stability of the numerical scheme. This is based on the use of two operators on each element: (a) a projection $\Pi^{\nabla}$, related to the bi-linear form of the problem, and (b) the $L^{2}$-projection $\Pi^{0}$ useful to address the time-dependent term. The local stiffness and mass matrices at the elemental level will be the sum of two matrices, one assuring consistency and the other one stability. For the numerical details in the computation of such matrices, the reader can refer to da Veiga et al. (2014); Sutton (2016). The VEM discretization leads to a system of non-linear ordinary differential equations:

$$
H(\mathbf{p}) \mathbf{p}+P(\mathbf{p}) \frac{d \mathbf{p}}{d t}+\mathbf{f}(\mathbf{p})=0
$$

which is numerically integrated by a backward Euler method in time and a fixed-point iteration scheme. Note that the right-hand side $\mathbf{f}(\mathbf{p})$ accounts for both boundary conditions and external forcing functions, i.e., the source term $c_{b}\left(\sigma_{z}\right)\left(1-\phi_{0}\right)\left(\gamma_{s}-\gamma\right) \omega(x, t)$ (Equation 7).

The numerical solution procedure is implemented as follows (Zoccarato and Teatini, 2017). First, the sedimentation thickness is computed accounting for the material deposited during the time step $\Delta t$, with the total stress $\sigma_{t}$ updated accordingly. Then, the solution $\mathbf{p}$ at the current time step is computed through a fixed-point iteration. At the $m$-th linear step of the non-linear scheme, the solution $\mathbf{p}^{(m)}$ is used to update $\sigma_{z}^{(m)}=\sigma_{t}-p^{(m)}$ keeping $\sigma_{t}$ constant within each time step. Using $\sigma_{z}^{(m)}$ and the constitutive relationship of the porous medium, the parameters $k_{x}\left(\sigma_{z}^{(m)}\right), k_{z}\left(\sigma_{z}^{(m)}\right), \phi\left(\sigma_{z}^{(m)}\right), \alpha\left(\sigma_{z}^{(m)}\right)$, and $c_{b}\left(\sigma_{z}^{(m)}\right)$ are also updated. The medium compaction is then computed through equation (4), stretching the grid node positions to start a new iteration. After convergence, i.e., $\left\|\mathbf{p}^{(m-1)}-\mathbf{p}^{(m)}\right\|<\tau$ for some user-specified tolerance $\tau$, the mesh is finally adjusted and, if necessary, new elements are added on the top of the marsh.

\section{MODEL SET UP AND RESULTS}

The proposed numerical scheme is employed to investigate the effectiveness of the methodology using two simple test cases. At the beginning of the simulations, a $2 \mathrm{D}$ section of a salt marsh is schematically represented by a rectangular domain (Figure 1). The grid extends for $1.50 \mathrm{~m}$ along the $x$-coordinate and is $0.15 \mathrm{~m}$-deep, with 10 uniform layers $0.015 \mathrm{~m}$-thick. The domain is initially discretized into a structured triangular grid consisting of 1650 nodes and 2980 elements, as shown in Figure 1. The mesh evolves in time by updating the node positions and adding new elements because of the sediment deposition on the top of the marsh. For the sake of simplicity, the marsh is here considered as a homogenous porous material characterized by geomechanical properties that vary with the inter-granular vertical effective stress according to a linear law. The initial porosity of the fresh material is 0.981 with a compressibility equal to $6.2 \cdot 10^{-5} \mathrm{kPa}^{-1}$. The soil grains are assumed incompressible with specific weight $\gamma_{s}=20.2 \mathrm{kN}$. The hydraulic conductivity field is uniform and isotropic with $k_{x}=k_{z}=1.0 \cdot 10^{-7} \mathrm{~m} / \mathrm{s}$. The boundary conditions are prescribed such that Dirichlet null values, i.e., hydrostatic pressure, are set on the top and on left boundary of the domain to simulate saturated condition on the marsh surface and the presence of a tidal creek at the marsh left edge. The basement 


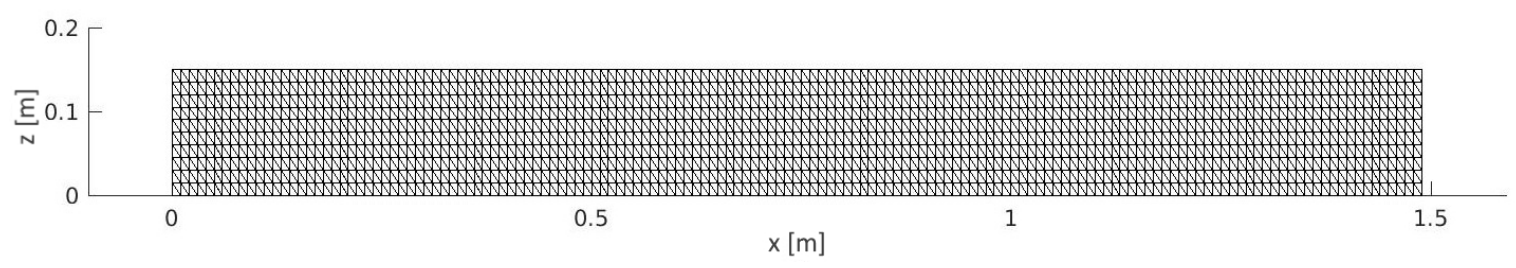

Figure 1. Initial mesh at time $t_{i}=0 \mathrm{yr}$ for both test cases.

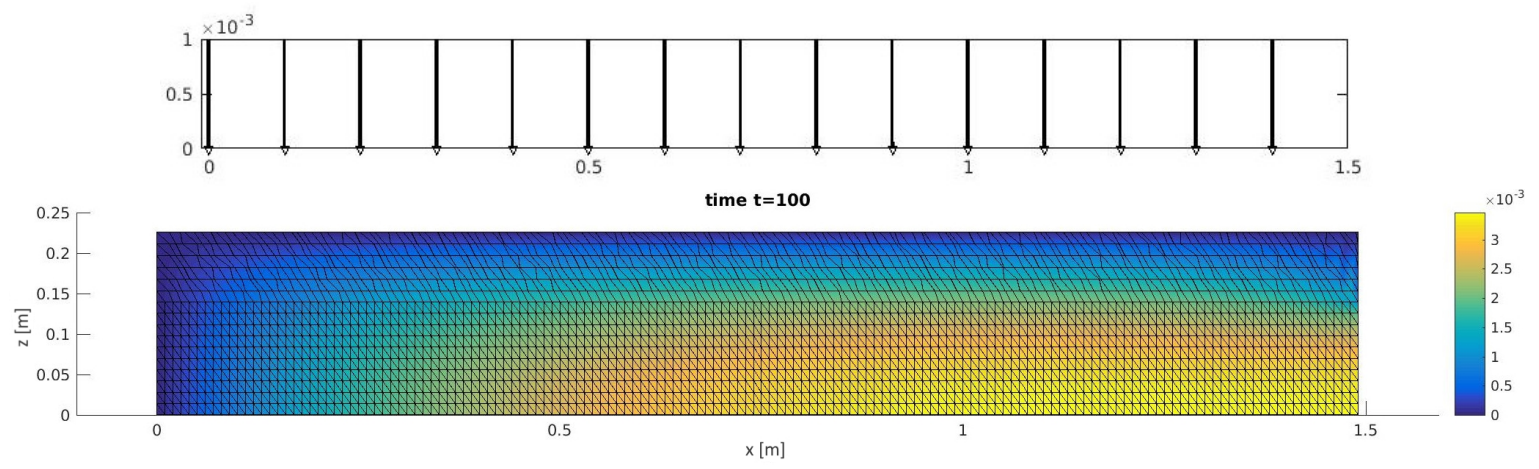

Figure 2. Mesh and $p$ distribution at $t_{f}=100$ yrs for the uniform load $\omega_{1}$.

is assumed impermeable and any in and/or out fluxes are prevented. The right edge of the domain is a no-flux boundary representing a symmetry axis for the marsh. Two different distributions of the sedimentation rate are considered for testing purposes. In the first case, a uniform function $\omega(x, t)$ is considered with a constant value $\omega_{1}=0.001 \mathrm{~m} / \mathrm{yr}$, whereas, in the second test, $\omega(x, t)$ is a function of space and time varying according to the exponential law $\omega_{2}=\omega_{1} e^{-x / l_{c}}$ where $l_{c}=0.04 \mathrm{~m}$. The time discretization is $\Delta t=1 \mathrm{yr}$ from $t_{i}=0$ yr up to the final time $t_{f}=100 \mathrm{yrs}$.

In the first test case, at each time time step, a constant value of the sedimentation thickness $z=\omega \cdot \Delta t$ is computed and added at each node of the marsh surface. Then, these values are compared with a maximum sedimentation thickness $z_{\max }$. If the computed $z$ is less than $z_{\max }$, the value of the thickness of each element is updated (by updating the $z$-coordinates of each nodal value), otherwise, new elements are added on the top of the marsh domain. To verify the potential of the VEM approach, new nodes and new elements are constructed in such a way that irregular triangles and quadrangles are added to the uniform triangular mesh. In particular, the position of every new node is perturbed by a random value, so that the newly generated elements have a random shape. Notice that initially the VEM solution on the triangular grid coincides with the FEM outcome. With the introduction of the new irregular elements, the VEM outcome still displays a smooth and accurate profile, even on general polygonal elements. Figure 2 shows the sedimentation rate $\omega_{1}$ and the overpressure $p$ at $t_{f}$. The number of nodes and elements increase to 2550 and 3880, respectively. The uniform sedimentation leads to a uniform surface elevation from $0.15 \mathrm{~m}$ to $0.23 \mathrm{~m}$ with an overall compaction of 0.02 $\mathrm{m}$. The second test represents a more realistic configuration where the inflow of inorganic sediments from the tidal creek into the marsh surface is simulated. The typical sedimentation rate has a spatial exponential decay as shown in Figure 3. The maximum overpressure is lower than in the previous case although the pressure distribution within the domain is quite similar. The number of nodes and elements increases up to 1843 and 3173 at $t_{f}$ with a maximum marsh height of $0.23 \mathrm{~m}$ at left edge. On the marsh right edge, no elements are added because $z$ is always lower than the threshold $z_{\max }$.

As highlighted in Figure 4, the zoom of the upper-left mesh of Figure 3 shows a grid with the simultaneous presence of both triangular and quadrangular elements. Moreover, these elements are expressly distorted along the $x$-coordinate to stress the potentiality of the VEM approach on general polygonal grids. Figure 4 also shows the differential compaction undergoing the grid elements. In particular, the element deformation diminishes from left-to-right edges due to the decreasing of the loading conditions. 

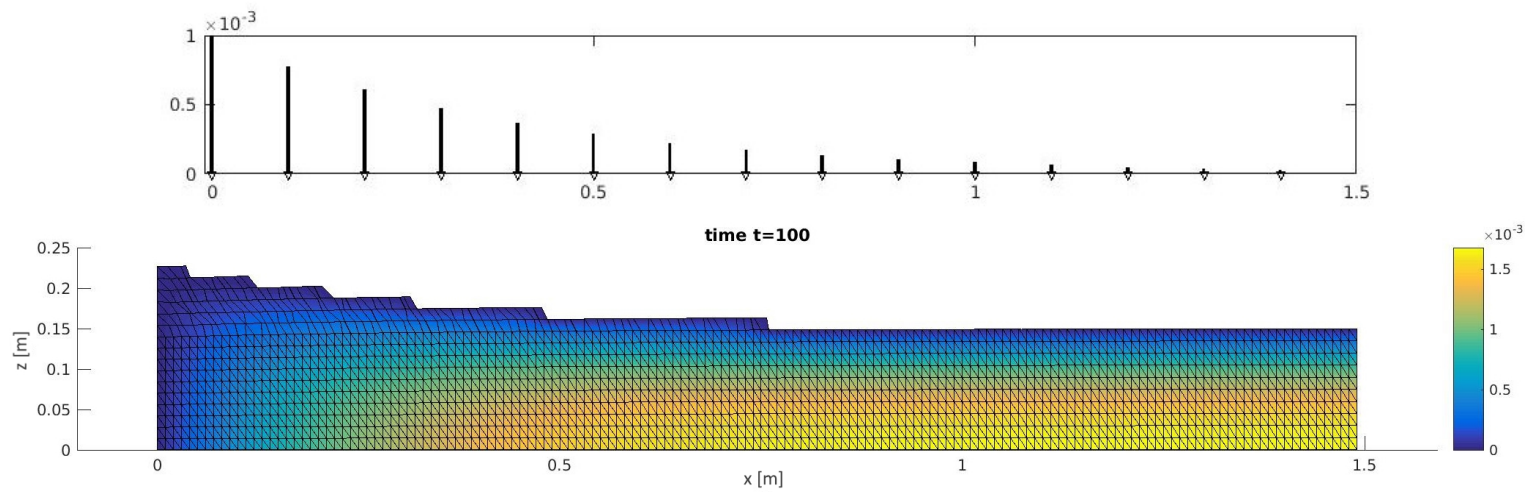

Figure 3. Mesh and $p$ distribution at $t_{f}=100 \mathrm{yrs}$ for the exponential load $\omega_{2}(x, t)$.

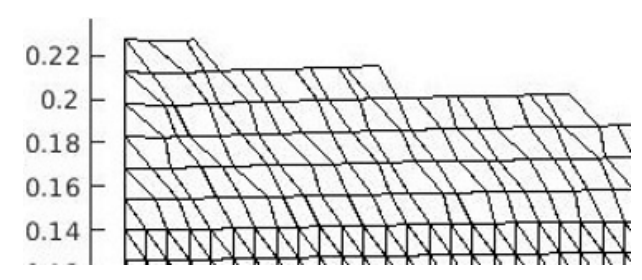

Figure 4. Zoom of the grid obtained in the second case at time $t=100 \mathrm{yrs}$.

Although the outcome of these test cases is quite preliminary, such applications provide evidence that the VEM approach well performs on irregular grids, allowing for a great flexibility in the discretization of any domain and avoiding problems related to mesh distortions. The solution in terms of overpressure in not affected by the element shape and the numerical accuracy is of the same order as the one obtained with the FEM approach on a regular triangular mesh (not shown here).

\section{CONCLUSIONS}

In this paper, the vertical dynamics of a marsh surface is investigated by using a 2D groundwater flow model coupled with a 1D geomechanical module discretized using the Virtual Element Method. This discretization technique appears to be particularly promising in this application as compared to the standard FEM due to its flexibility in the element generation and management. Due to the high porosity and compressibility of the newly-deposited material, the model takes also into account the geometric non-linearity caused by the large salt marsh deformations. The use of a VEM approach potentially avoids the numerical problems arising from strongly distorted meshes.

Two test cases are carried out to show the possible applications of the proposed methodology. The tests differ for the space and time distribution of sedimentation rate over the marsh surface. The results show a possible construction of an adaptive mesh with distorted elements, but not affecting the numerical solution. The use of VEM is encouraging in applications where the mesh should adapt to the evolution of a dynamic system, avoiding expensive re-meshing procedures at each time step, such as those implemented by Zoccarato and Teatini (2017), with an overall reduction of the computational cost. Further developments are expected when the material heterogeneity is introduced in the modeling framework to simulate the behavior of soils with different hydro-geomechanical properties.

\section{ACKNOWLEDGEMENTS}

This research was funded by the Flagship Project RITMARE - The Italian Research for the Sea, CNR-MIUR, National Research Program 2011-2013, "Linea SOLVE”. 
M. Ferronato et al., A Virtual Element Model for the prediction ...

\section{REFERENCES}

Allen, J. R. L. (1999). Geological impact on coastal wetland landscapes: some general effects of sediment autocompaction in the holocene of northwest europe. Holocene 9(1), 1-12.

Allen, J. R. L. (2000). Morphodynamics of holocene salt marshes: a review sketch from the atlantic and southern north sea coasts of europe. Quaternary Sci. Rev. 19, 1155-1231.

Brain, M. J., A. J. Long, D. N. Petley, B. P. Horton, and R. J. Allison (2011). Compression behaviour of minerogenic low energy intertidal sediments. Sed. Geol. 233(1-4), 28-41.

da Veiga, L. B., F. Brezzi, A. Cangiani, G. Manzini, L. D. Marini, and A. Russo (2013). Basic principles of Virtual Element Methods. Mathematical Models and Methods in Applied Sciences 23(1), 199-214.

da Veiga, L. B., F. Brezzi, L. D. Marini, and A. Russo (2014). The hitchhiker's guide to the Virtual Element Method. Mathematical Models and Methods in Applied Sciences 24(8), 1541-1573.

Gambolati, G. (1973a). Equation for one-dimensional vertical flow of groundwater. 1. The rigorous theory. Water Resour. Res. 9(4), 1022-1028.

Gambolati, G. (1973b). Equation for one-dimensional vertical flow of groundwater. 2. Validity range of the diffusion equation. Water Resour. Res. 9(5), 1385-1395.

Gambolati, G., G. Giunta, and P. Teatini (1998). Numerical modeling of natural land subsidence over sedimentary basins undergoing large compaction. In G. Gambolati (Ed.), CENAS - Coastiline evolution of the Upper Adriatic Sea due to sea level rise and natural and anthropogenic land subsidence, Number 28 in Water Science and Technology Library, pp. 77-102. Klwer Acedemic Publ.

Kooi, H. and J. J. de Vries (1998). Land subsidence and hydrodynamic compaction of sedimentary basin. Hydrol. Earth System. Sci. 2(1-2), 159-171.

Massey, A. C., M. A. Paul, W. R. Gehrels, and D. J. Charman (2006). Autocompaction in Holocene coastal back-barrier sediments from south Devon, southwest England, UK. Mar. Geol. 226(3-4), 225-241.

Morris, J. T., P. V. Sundareshwar, C. T. Nietch, B. Kjerfve, and D. R. Cahoon (2002). Response of coastal wetlands to rising sea level. Ecology 83(10), 2869-2877.

Paul, M. A. and B. F. Barras (1998). A geotechnical correction for post-depositional sediment compression: examples from the Forth valley, Scotland. J. Quaternary Sci. 13(2), 171-176.

Pizzuto, J. E. and A. E. Schwendt (1997). Mathematical modeling of autocompaction of a holocene transgressive valley-fill deposit, Wolfe Glade, Delaware. Geology 25(1), 57-60.

Sheldon, N. D. and G. J. Retallack (2001). Equation for compaction of paleosols due to burial. Geology 29(3), 247-250.

Skempton, A. W. (1970). The consolidation of clays by gravitational compaction. Q. J. Geol. Soc. 125(1-4), 373-411.

Sutton, O. J. (2016). The virtual element method in 50 lines of MATLAB. Numerical Algorithms in press.

Zoccarato, C. and P. Teatini (2017). Numerical simulations of holocene salt-marsh dynamics under the hypothesis of large soil deformations. Advances in Water Resources (Under Review). 